\title{
Editorial
}

Neuro

epidemiology

\section{A Commentary on the Standards of Reporting of Neurological Disorders Checklist: A Guideline for the Reporting of Descriptive Studies in Neuroepidemiology}

\author{
Derrick A. Bennett ${ }^{a}$ Carol Brayne ${ }^{b}$ Valery Feigin ${ }^{c}$ \\ ${ }^{a}$ Nuffield Department of Population Health, University of Oxford, Oxford, ${ }^{b}$ Department of Public Health and \\ Primary Care, University of Cambridge, Cambridge, UK; ' National Institute for Stroke and Applied Neurosciences, \\ AUT University, Auckland, New Zealand
}

Neurological diseases are becoming more prevalent as the world's population ages, and their burden is expected to increase globally [1]. These conditions are often subtle in their clinical manifestation and are prone to misconceptions and misinterpretations [2]. In epidemiological studies, neurological conditions can provide particular challenges such as rare conditions, variable diagnostic criteria that may also need sophisticated technology, and different stages of the disease. High-quality prevalence and incidence studies of neurological conditions that follow a systematic approach are essential for estimating the burden of disease globally for comparing the estimates between various countries and populations, for priority setting, resource allocation and for planning public health approaches. For neurological conditions, descriptive epidemiological studies can provide important information on (a) trends and gaps in the health service needs; (b) estimates of morbidity, mortality and economic burden from these diseases; and (c) can be used for generating new hypotheses on causation or natural history of the disease. Descriptive epidemiological studies are particularly useful for estimating prevalence, incidence, morbidity and mortality time trends for studies where global health is of concern [3]. We developed a reporting guideline using a consensus-based approach [4-6] that outlines the key information to be reported for descriptive health pol- (c) 2015 S. Karger AG, Basel

0251-5350/15/0452-0071\$39.50/0 icy research, (such as Global Burden of Disease and Injuries type studies (GBD)) [7]. This was because studies that are not necessarily obviously population based, particularly those from settings where there are few data, might be of greater value to projects such as the GBD that needs to synthesise evidence, if they were reported better. The Standards of Reporting of Neurological Disorders (STROND) checklist has 15 key items and distinguishes between items that are deemed to be 'basic minimum reporting' requirements and items that are deemed to be 'ideal reporting' requirements. Complete details on how the STROND checklist was designed and developed have been reported elsewhere [8].

It is envisaged that although the STROND checklist is aimed at improving the reporting of descriptive epidemiological studies of neurological disorders, we believe that it would also be of interest to researchers wishing to report their descriptive epidemiological studies in other areas. The 15 -item checklist provides a framework to satisfy the need for completeness and transparency of reporting of incidence and prevalence studies of neurological disorders. We attempted to strike a balance between the adequate detail and concise reporting, so that we incorporate both 'basic minimum reporting' standards as well as 'ideal reporting' criteria in the checklist. As has been done with other reporting guidelines [9, 
10], we aim to produce an explanation and elaboration report that provides more detail on the rationale for each item included in the checklist as well as some empirical evidence of good reporting of incidence and prevalence studies for neurological disorders. The ultimate goal is that the use of the STROND checklist will lead to more consistent, transparent and contextualised reporting of clinical studies, case series and all research from which generalizable population data might be derived. We hope that these findings, which are more applicable will lead to better healthcare decisions in the future.

\section{References}

1 Whiteford HA, Ferrari AJ, Degenhardt L, Feigin V, Vos T: The global burden of mental, neurological and substance use disorders: an analysis from the global burden of disease study 2010. PLoS One 2015;10:e0116820.

-2 Rao D, Choi SW, Victorson D, Bode R, Peterman A, Heinemann A, et al: Measuring stigma across neurological conditions: the development of the stigma scale for chronic illness (SSCI). Qual Life Res 2009;18:585-595.

-3 Murray CJ, Ezzati M, Flaxman AD, Lim S, Lozano R, Michaud C, et al: GBD 2010: a multiinvestigator collaboration for global comparative descriptive epidemiology. Lancet 2012; 380:2055-2058.
4 Yancik R, Maklan CW: The Delphi method as a research tool in gerontological curriculum planning. Gerontol Geriatr Educ 1981;1:163168.

5 Steurer J: The Delphi method: an efficient procedure to generate knowledge. Skeletal Radiol 2011;40:959-961.

6 Wang X, Li W: [Delphi method and its use in clinical medicine]. Zhonghua Wai Ke Za Zhi 2014;52:7-10.

7 Chin JH, Vora N: The global burden of neurologic diseases. Neurology 2014;83:349-351.

$>8$ Bennett DA, Brayne C, Feigin VL, BarkerCollo S, Brainin M, Davis D, et al: Development of the standards of reporting of neuro- logical disorders (STROND) checklist: a guideline for the reporting of incidence and prevalence studies in neuroepidemiology. Eur J Epidemiol 2015;30:569-576.

9 Moher D, Hopewell S, Schulz KF, Montori V, Gøtzsche PC, Devereaux PJ, et al: CONSORT 2010 explanation and elaboration: updated guidelines for reporting parallel group randomised trials. BMJ 2010;340:c869.

10 Moons KG, Altman DG, Reitsma JB, Ioannidis JP, Macaskill P, Steyerberg EW, et al: Transparent reporting of a multivariable prediction model for individual prognosis or diagnosis (TRIPOD): the TRIPOD statement. Ann Intern Med 2015;162:W1-W73. 